Commun. Korean Math. Soc. 28 (2013), No. 2, pp. 231-241

http://dx.doi.org/10.4134/CKMS.2013.28.2.231

\title{
A COUNTEREXAMPLE FOR IMPROVED SOBOLEV INEQUALITIES OVER THE 2-ADIC GROUP
}

\author{
Diego Chamorro
}

\begin{abstract}
On the framework of the 2 -adic group $\mathbb{Z}_{2}$, we study a Sobolevlike inequality where we estimate the $L^{2}$ norm by a geometric mean of the $B V$ norm and the $\dot{B}_{\infty}^{-1, \infty}$ norm. We first show, using the special topological properties of the $p$-adic groups, that the set of functions of bounded variations $B V$ can be identified to the Besov space $\dot{B}_{1}^{1, \infty}$. This identification lead us to the construction of a counterexample to the improved Sobolev inequality.
\end{abstract}

\section{Introduction}

The general improved Sobolev inequalities were initially introduced by P. Grard, Y. Meyer and F. Oru in [6]. For a function $f$ such that $f \in \dot{W}^{s_{1}, p}\left(\mathbb{R}^{n}\right)$ and $f \in \dot{B}_{\infty}^{-\beta, \infty}\left(\mathbb{R}^{n}\right)$, these inequalities read as follows:

$$
\|f\|_{\dot{W}^{s, q}} \leq C\|f\|_{\dot{W}^{s_{1}, p}}^{\theta}\|f\|_{\dot{B}_{\infty}^{-\beta, \infty}}^{1-\theta}
$$

where $1<p<q<+\infty, \theta=p / q, s=\theta s_{1}-(1-\theta) \beta$ and $-\beta<s<s_{1}$. The method used for proving these estimates relies on the Littlewood-Paley decomposition and on a dyadic bloc manipulation and this explains the fact that the value $p=1$ is forbidden here.

In order to study the case $p=1$, it is necessary to develop other techniques. The case when $p=1, s=0$ and $s_{1}=1$ was treated by M. Ledoux in [11] using a special cut-off function; while the case $s_{1}=1$ and $p=1$ was studied by A. Cohen, W. Dahmen, I. Daubechies and R. De Vore in [5]. In this last article, the authors give a BV-norm weak estimation using wavelet coefficients and isoperimetric inequalities and obtained, for a function $f$ such that $f \in B V\left(\mathbb{R}^{n}\right)$ and $f \in \dot{B}_{\infty}^{-\beta, \infty}\left(\mathbb{R}^{n}\right)$, the estimation below:

$$
\|f\|_{\dot{W}^{s, q}} \leq C\|f\|_{B V}^{1 / q}\|f\|_{\dot{B}_{\infty}^{-\beta, \infty}}^{1-1 / q}
$$

where $1<q \leq 2,0 \leq s<1 / q$ and $\beta=(1-s q) /(q-1)$.

Received November 8, 2010; Revised November 12, 2012.

2010 Mathematics Subject Classification. 22E35, 46E35.

Key words and phrases. Sobolev inequalities, $p$-adic groups. 
In a previous work (see [3], [4]), we studied the possible generalizations of inequalities of type (1) and (2) to other frameworks than $\mathbb{R}^{n}$. In particular, we worked over stratified Lie groups and over polynomial volume growth Lie groups and we obtained some new weak-type estimates.

The aim of this paper is to study inequalities of type (1) and (2) in the setting of the 2 -adic group $\mathbb{Z}_{2}$. The main reason for working in the framework of $\mathbb{Z}_{2}$ is that this group is completely different from $\mathbb{R}^{n}$ and from stratified or polynomial Lie groups. Indeed, since the 2-adic group is totally discontinuous, it is not absolutely trivial to give a definition for smoothness measuring spaces. Thus, the first step to do, in order to study these Sobolev-like inequalities, is to give an adapted characterization of such functional spaces. In the present article, this will be achieved using the Littlewood-Paley approach and, once this task is done, we will immediatly prove -following the classical path exposed in [6]- the inequalities (1) in the setting of the 2 -adic group $\mathbb{Z}_{2}$.

For the estimate (2), we introduce the $B V$ space in the following manner: we will say that $f \in B V\left(\mathbb{Z}_{2}\right)$ if there exists a constant $C>0$ such that

$$
\int_{\mathbb{Z}_{2}}|f(x+y)-f(x)| d x \leq C|y|_{2} \quad\left(\forall y \in \mathbb{Z}_{2}\right) .
$$

As a surprising fact, we obtain the follwing.

Theorem 1. We have the following relationship between the space of functions of bounded variation $B V\left(\mathbb{Z}_{2}\right)$ and the Besov space $\dot{B}_{1}^{1, \infty}\left(\mathbb{Z}_{2}\right)$ :

$$
B V\left(\mathbb{Z}_{2}\right) \simeq \dot{B}_{1}^{1, \infty}\left(\mathbb{Z}_{2}\right) .
$$

Of course, this identification is false in $\mathbb{R}^{n}$ and it is this special relationship in $\mathbb{Z}_{2}$ that give us our principal theorem which is the 2-adic counterpart of the inequality (2):

Theorem 2. The following inequality is false in $\mathbb{Z}_{2}$. There is not an universal constant $C>0$ such that we have

$$
\|f\|_{L^{2}}^{2} \leq C\|f\|_{B V}\|f\|_{\dot{B}_{\infty}^{-1, \infty}}
$$

for all $f \in B V \cap \dot{B}_{\infty}^{-1, \infty}\left(\mathbb{Z}_{2}\right)$.

This striking fact says that the improved Sobolev inequalities of type (2) depend on the group's structure and that they are no longer true for the 2-adic group $\mathbb{Z}_{2}$.

The plan of the article is the following: in Section 2 we recall some well known properties about $p$-adic groups, in Section 3 we define Sobolev and Besov spaces, in Section 4 we prove Theorem 1 and, finally, we prove Theorem 2 in Section 5.

Before finishing these preliminary remarks, it is important to say that the inequality (1) was generalized in [13] where the Besov space $\dot{B}_{\infty}^{-\beta, \infty}$ is replaced by the $B M O$ space. Thus, with the study of $p$-adic $B M O$ functions given in 
[7] and [8] it would be interesting to investigate if such generalization is still valid in a $p$-adic setting.

\section{2. $p$-adic groups}

We write $a \mid b$ when $a$ divide $b$ or, equivalently, when $b$ is a multiple of $a$. Let $p$ be any prime number, for $0 \neq x \in \mathbb{Z}$, we define the $p$-adic valuation of $x$ by $\gamma(x)=\max \left\{r: p^{r} \mid x\right\} \geq 0$ and, for any rational number $x=\frac{a}{b} \in \mathbb{Q}$, we write $\gamma(x)=\gamma(a)-\gamma(b)$. Furthermore if $x=0$, we agree to write $\gamma(0)=+\infty$.

Let $x \in \mathbb{Q}$ and $p$ be any prime number, with the $p$-adic valuation of $x$ we can construct a norm by writing

$$
|x|_{p}= \begin{cases}p^{-\gamma} & \text { if } \quad x \neq 0 \\ p^{-\infty}=0 & \text { if } \quad x=0 .\end{cases}
$$

This expression satisfy the following properties

a) $|x|_{p} \geq 0$, and $|x|_{p}=0 \Longleftrightarrow x=0$;

b) $|x y|_{p}=|x|_{p}|y|_{p}$;

c) $|x+y|_{p} \leq \max \left\{|x|_{p},|y|_{p}\right\}$, with equality when $|x|_{p} \neq|y|_{p}$.

When a norm satisfy $c$ ) it is called a non-Archimedean norm and an interesting fact is that over $\mathbb{Q}$ all the possible norms are equivalent to $|\cdot|_{p}$ for some $p$ : this is the so-called Ostrowski theorem (see [1] for a proof).

Definition 2.1. Let $p$ be any prime number. We define the field of $p$-adic numbers $\mathbb{Q}_{p}$ as the completion of $\mathbb{Q}$ when using the norm $|\cdot|_{p}$.

We present in the following lines the algebraic structure of the set $\mathbb{Q}_{p}$. Every $p$-adic number $x \neq 0$ can be represented in a unique manner by the formula

$$
x=p^{\gamma}\left(x_{0}+x_{1} p+x_{2} p^{2}+\cdots\right),
$$

where $\gamma=\gamma(x)$ is the $p$-adic valuation of $x$ and $x_{j}$ are integers such that $x_{0}>0$ and $0 \leq x_{j} \leq p-1$ for $j=1,2, \ldots$. Remark that this canonical representation implies the identity $|x|_{p}=p^{-\gamma}$.

Let $x, y \in \mathbb{Q}_{p}$, using the formula (4) we define the sum of $x$ and $y$ by $x+y=p^{\gamma(x+y)}\left(c_{0}+c_{1} p+c_{2} p^{2}+\cdots\right)$ with $0 \leq c_{j} \leq p-1$ and $c_{0}>0$, where $\gamma(x+y)$ and $c_{j}$ are the unique solution of the equation

$$
\begin{aligned}
& p^{\gamma(x)}\left(x_{0}+x_{1} p+x_{2} p^{2}+\cdots\right)+p^{\gamma(y)}\left(y_{0}+y_{1} p+y_{2} p^{2}+\cdots\right) \\
= & p^{\gamma(x+y)}\left(c_{0}+c_{1} p+c_{2} p^{2}+\cdots\right) .
\end{aligned}
$$

Furthermore, for $a, x \in \mathbb{Q}_{p}$, the equation $a+x=0$ has a unique solution in $\mathbb{Q}_{p}$ given by $x=-a$. In the same way, the equation $a x=1$ has a unique solution in $\mathbb{Q}_{p}: x=1 / a$.

We take now a closer look at the topological structure of $\mathbb{Q}_{p}$. With the norm $|\cdot|_{p}$ we construct a distance over $\mathbb{Q}_{p}$ by writing

$$
d(x, y)=|x-y|_{p}
$$


and we define the balls $B_{\gamma}(x)=\left\{y \in \mathbb{Q}_{p}: d(x, y) \leq p^{\gamma}\right\}$ with $\gamma \in \mathbb{Z}$. Remark that, from the properties of the $p$-adic valuation, this distance has the ultrametric property (i.e., $\left.d(x, y) \leq \max \{d(x, z), d(z, y)\} \leq|x|_{p}+|y|_{p}\right)$.

We gather with the next proposition some important facts concerning the balls in $\mathbb{Q}_{p}$.

Proposition 2.1. Let $\gamma$ be an integer, then we have

1) the ball $B_{\gamma}(x)$ is an open and a closed set for the distance (5).

2) every point of $B_{\gamma}(x)$ is its center.

3) $\mathbb{Q}_{p}$ endowed with this distance is a complete Hausdorff metric space.

4) $\mathbb{Q}_{p}$ is a locally compact set.

5) the p-adic group $\mathbb{Q}_{p}$ is a totally discontinuous space. [14].

For a proof of this proposition and more details see the books [1], [10] or

\section{Functional spaces}

In this article, we will work with the subset $\mathbb{Z}_{2}$ of $\mathbb{Q}_{2}$ which is defined by $\mathbb{Z}_{2}=\left\{x \in \mathbb{Q}_{2}:|x|_{2} \leq 1\right\}$, and we will focus on real-valued functions over $\mathbb{Z}_{2}$. Since $\mathbb{Z}_{2}$ is a locally compact commutative group, there exists a Haar measure $d x$ which is translation invariant i.e., $d(x+a)=d x$, furthermore we have the identity $d(x a)=|a|_{2} d x$ for $a \in \mathbb{Z}_{2}^{*}$. We will normalize the measure $d x$ by setting

$$
\int_{\left\{|x|_{2} \leq 1\right\}} d x=1
$$

This measure is then unique and we will note $|E|$ the measure for any subset $E$ of $\mathbb{Z}_{2}$.

Another type of measures can be considered on the $p$-adic setting (see for example [9]). However, in this article we will only work with the previous one.

Lebesgue spaces $L^{p}\left(\mathbb{Z}_{2}\right)$ are thus defined in a natural way: $\|f\|_{L^{p}}=\left(\int_{\mathbb{Z}_{2}}|f(x)|^{p} d x\right)^{1 / p}$ for $1 \leq p<+\infty$, with the usual modifications when $p=+\infty$.

Let us now introduce the Littlewood-Paley decomposition in $\mathbb{Z}_{2}$. We note $\mathcal{F}_{j}$ the Boole algebra formed by the equivalence classes $E \subset \mathbb{Z}_{2}$ modulo the subgroup $2^{j} \mathbb{Z}_{2}$. Then, for any function $f \in L^{1}\left(\mathbb{Z}_{2}\right)$, we call $S_{j}(f)$ the conditionnal expectation of $f$ with respect to $\mathcal{F}_{j}$ :

$$
S_{j}(f)(x)=\frac{1}{\left|B_{j}(x)\right|} \int_{B_{j}(x)} f(y) d y .
$$

The dyadic blocks are thus defined by the formula $\Delta_{j}(f)=S_{j+1}(f)-S_{j}(f)$ and the Littlewood-Paley decomposition of a function $f: \mathbb{Z}_{2} \longrightarrow \mathbb{R}$ is given by

$$
f=S_{0}(f)+\sum_{j=0}^{+\infty} \Delta_{j}(f) \quad \text { where } S_{0}(f)=\int_{\mathbb{Z}_{2}} f(x) d x .
$$


We will need in the sequel some very special sets noted $Q_{j, k}$. Here is the definition and some properties:

Proposition 3.1. Let $j \in \mathbb{N}$ and $k=\left\{0,1, \ldots, 2^{j}-1\right\}$. Define the subset $Q_{j, k}$ of $\mathbb{Z}_{2}$ by

$$
Q_{j, k}=\left\{k+2^{j} \mathbb{Z}_{2}\right\}
$$

Then

1) We have the identity $\mathcal{F}_{j}=\bigcup_{0 \leq k<2^{j}} Q_{j, k}$,

2) For $k=\left\{0,1, \ldots, 2^{j}-1\right\}$ the sets $Q_{j, k}$ are mutually disjoint,

3) $\left|Q_{j, k}\right|=2^{-j}$ for all $k$,

4) the 2-adic valuation is constant over $Q_{j, k}$.

The verifications are easy and left to the reader.

With the Littlewood-Paley decomposition given in (6), we obtain the following equivalence for the Lebesgue spaces $L^{p}\left(\mathbb{Z}_{2}\right)$ with $1<p<+\infty$ :

$$
\|f\|_{L^{p}} \simeq\left\|S_{0}(f)\right\|_{L^{p}}+\left\|\left(\sum_{j \in \mathbb{N}}\left|\Delta_{j} f\right|^{2}\right)^{1 / 2}\right\|_{L^{p}} .
$$

See the book [12], Chapter IV, for a general proof.

Let us turn now to smoothness measuring spaces. As said in the introduction, it is not absolutely trivial to define Sobolev and Besov spaces over $\mathbb{Z}_{2}$ since we are working in a totally discontinuous setting. Here is an example of this situation with the Sobolev space $W^{1,2}$ : one could try to define the quantity $|\nabla f|$ by the formula

$$
|\nabla f|=\lim _{\delta \rightarrow 0} \sup _{d(x, y)<\delta} \frac{|f(x)-f(y)|}{d(x, y)}
$$

and define the Sobolev space $W^{1,2}\left(\mathbb{Z}_{2}\right)$ by the norm

$$
\|f\|_{*}=\|f\|_{L^{2}}+\left(\int_{\mathbb{Z}_{2}}|\nabla f|^{2} d x\right)^{1 / 2} .
$$

Now, using the Littlewood-Paley decomposition we can also write

$$
\|f\|_{* *}=\left\|S_{0} f\right\|_{L^{2}}+\left\|\left(\sum_{j \in \mathbb{N}} 2^{2 j}\left|\Delta_{j} f\right|^{2}\right)^{1 / 2}\right\|_{2} .
$$

However, the quantities $\|\cdot\|_{*}$ and $\|\cdot\|_{* *}$ are not equivalent: in the case of (8) consider a function $f=c_{k}$ constant over each $Q_{j, k}=\left\{k+2^{j} \mathbb{Z}_{2}\right\}$ for some fixed $j$. Then we have $|\nabla f| \equiv 0$ and for these functions the norm $\|\cdot\|_{*}$ would be equal to the $L^{2}$ norm. 
This is the reason why we will use in this article the Littlewood-Paley approach to characterize Sobolev spaces:

$$
\|f\|_{W^{s, p}} \simeq\left\|S_{0} f\right\|_{L^{p}}+\left\|\left(\sum_{j \in \mathbb{N}} 2^{2 j s}\left|\Delta_{j} f\right|^{2}\right)^{1 / 2}\right\|_{L^{p}},
$$

with $1<p<+\infty$ and $s>0$. For Besov spaces we will define them by the norm

$$
\|f\|_{B_{p}^{s, q}} \simeq\left\|S_{0} f\right\|_{L^{p}}+\left(\sum_{j \in \mathbb{N}} 2^{j s q}\left\|\Delta_{j} f\right\|_{L^{p}}^{q}\right)^{1 / q},
$$

where $s \in \mathbb{R}, 1 \leq p, q<+\infty$ with the necessary modifications when $p, q=+\infty$.

Remark 1. For homogeneous functional spaces $\dot{W}^{s, p}$ and $\dot{B}_{p}^{s, q}$, we drop out the term $\left\|S_{0} f\right\|_{L^{p}}$ in $(9)$ and $(10)$.

Let us give some simple examples of function belonging to these functional spaces.

1) The function $f(x)=\log _{2}|x|_{2}$ is in $\dot{B}_{1}^{1, \infty}\left(\mathbb{Z}_{2}\right)$. First note that $|x|_{2}=$ $2^{-\gamma(x)}$ and thus $f(x)=-\gamma(x)$. Recall (cf. Proposition 3.1) that over each set $Q_{j, k}$, the quantity $\gamma(x)$ is constant, so the dyadic bloc $\Delta_{j} f$ is given by

$$
\Delta_{j} f(x)= \begin{cases}-1 & \text { over } Q_{j+1,0} \\ 0 & \text { elsewhere. }\end{cases}
$$

Hence, taking the $L^{1}$ norm, we have $\left\|\Delta_{j} f\right\|_{L^{1}}=\frac{1}{2} 2^{-j}$ and then $f \in$ $\dot{B}_{1}^{1, \infty}\left(\mathbb{Z}_{2}\right)$.

2) Set $h(x)=1 /|x|_{2}$, we have $h \in \dot{B}_{\infty}^{-1, \infty}$. For this, we must verify $\sup _{j \geq 0} 2^{-j}\left\|\Delta_{j} h\right\|_{L^{\infty}}<+\infty$. By definition we obtain $h(x)=2^{\gamma(x)}$ and $j \geq 0$

then

$$
\Delta_{j} h(x)= \begin{cases}2^{j} & \text { over } Q_{j+1,0} \\ 0 & \text { elsewhere. }\end{cases}
$$

We finally obtain $\left\|\Delta_{j} h\right\|_{L^{\infty}}=2^{j}$ and hence $2^{-j}\left\|\Delta_{j} h\right\|_{L^{\infty}}=1$ for all $j$, so we write $h \in \dot{B}_{\infty}^{-1, \infty}$.

With the Littlewood-Paley characterisation of Sobolev spaces and Besov spaces given in (9) and (10) we have the following theorem:

Theorem 3. In the framework of the 2-adic group $\mathbb{Z}_{2}$ we have, for a function $f$ such that $f \in \dot{W}^{s_{1}, p}\left(\mathbb{Z}_{2}\right)$ and $f \in \dot{B}_{\infty}^{-\beta, \infty}\left(\mathbb{Z}_{2}\right)$, the inequality

$$
\|f\|_{\dot{W}^{s, q}} \leq C\|f\|_{\dot{W}^{s_{1}, p}}^{\theta}\|f\|_{\dot{B}_{\infty}^{-\beta, \infty}}^{1-\theta}
$$

where $1<p<q<+\infty, \theta=p / q, s=\theta s_{1}-(1-\theta) \beta$ and $-\beta<s<s_{1}$. 
Proof. We start with an interpolation result: let $\left(a_{j}\right)_{j \in \mathbb{N}}$ be a sequence, let $s=\theta s_{1}-(1-\theta) \beta$ with $\theta=p / q$, then we have for $r, r_{1}, r_{2} \in[1,+\infty]$ the estimate

$$
\left\|2^{j s} a_{j}\right\|_{\ell^{r}} \leq C\left\|2^{j s_{1}} a_{j}\right\|_{\ell^{r_{1}}}^{\theta}\left\|2^{-j \beta} a_{j}\right\|_{\ell^{r_{2}}}^{1-\theta} .
$$

See [2] for a proof. Apply this estimate to the dyadic blocks $\Delta_{j} f$ to obtain

$$
\begin{aligned}
& \left(\sum_{j \in \mathbb{Z}} 2^{2 j s}\left|\Delta_{j} f(x)\right|^{2}\right)^{1 / 2} \\
\leq & C\left(\sum_{j \in \mathbb{Z}} 2^{2 j s_{1}}\left|\Delta_{j} f(x)\right|^{2}\right)^{\theta / 2}\left(\sup _{j \in \mathbb{Z}} 2^{-j \beta}\left|\Delta_{j} f(x)\right|\right)^{1-\theta} .
\end{aligned}
$$

To finish, compute the $L^{q}$ norm of the preceding quantities.

\section{The $B V\left(\mathbb{Z}_{2}\right)$ space and the proof of Theorem 1}

We study in this section the space of functions of bounded variation $B V$ and we will prove some surprising facts in the framework of 2-adic group $\mathbb{Z}_{2}$. Let us start recalling the definition of this space:

Definition 4.1. If $f$ is a real-valued function over $\mathbb{Z}_{2}$, we will say that $f \in$ $B V\left(\mathbb{Z}_{2}\right)$ if there exists a constant $C>0$ such that

$$
\int_{\mathbb{Z}_{2}}|f(x+y)-f(x)| d x \leq C|y|_{2}, \quad\left(\forall y \in \mathbb{Z}_{2}\right) .
$$

We prove now Theorem 1 which asserts that in $\mathbb{Z}_{2}$, the $B V$ space can be identified to the Besov space $\dot{B}_{1}^{1, \infty}$. For this, we will use two steps given by Propositions 4.1 and 4.2 below.

Proposition 4.1. If $f$ is a real-valued function over $\mathbb{Z}_{2}$ belonging to the Besov space $\dot{B}_{1}^{1, \infty}$, then $f \in B V$ and we have the inclusion $\dot{B}_{1}^{1, \infty} \subseteq B V$.

Proof. Let $f \in \dot{B}_{1}^{1, \infty}\left(\mathbb{Z}_{2}\right)$ and let us fix $|y|_{2}=2^{-m}$. We have to prove the following estimation for all $m>0$

$$
I=\int_{\mathbb{Z}_{2}}|f(x+y)-f(x)| d x \leq C 2^{-m} .
$$

Using the Littlewood-Paley decomposition given in (6), we will work on the formula below

$$
I=\left\|\left(S_{0} f(x+y)+\sum_{j \geq 0} \Delta_{j} f(x+y)\right)-\left(S_{0} f(x)+\sum_{j \geq 0} \Delta_{j} f(x)\right)\right\|_{L^{1}} .
$$

Then, by the dyadic block's properties we have to study

$$
I \leq\left\|S_{m} f(x+y)-S_{m} f(x)\right\|_{L^{1}}+\sum_{j=m+1}^{+\infty}\left\|\Delta_{j} f(x+y)-\Delta_{j} f(x)\right\|_{L^{1}} .
$$


We estimate this inequality with the two following lemmas.

Lemma 4.1. The first term in (12) is identically zero.

Proof. Since we have fixed $|y|_{2}=2^{-m}$, then for $x \in Q_{m, k}$, we have $x+y \in Q_{m, k}$ with $k=\left\{0, \ldots, 2^{m}-1\right\}$. Applying the operators $S_{m}$ to the functions $f(x+y)$ and $f(x)$ we get the desired result.

The second term in (12) is treated by the next lemma.

Lemma 4.2. Under the hypothesis of Proposition 4.1 and for $|y|_{2}=2^{-m}$ we have

$$
\sum_{j=m+1}^{+\infty}\left\|\Delta_{j} f(x+y)-\Delta_{j} f(x)\right\|_{L^{1}} \leq C 2^{-m}
$$

Proof. Indeed,

$$
\sum_{j=m+1}^{+\infty}\left\|\Delta_{j} f(x+y)-\Delta_{j} f(x)\right\|_{L^{1}} \leq 2 \sum_{j=m+1}^{+\infty}\left\|\Delta_{j} f\right\|_{L^{1}} .
$$

We use now the fact $\left\|\Delta_{j} f\right\|_{L^{1}} \leq C 2^{-j}$ for all $j$, since $f \in \dot{B}_{1}^{1, \infty}$, to get

$$
\sum_{j=m+1}^{+\infty}\left\|\Delta_{j} f(x+y)-\Delta_{j} f(x)\right\|_{L^{1}} \leq C 2^{-m} .
$$

With these two lemmas, and getting back to (12), we deduce the following inequality for all $y \in \mathbb{Z}_{2}$ :

$$
\int_{\mathbb{Z}_{2}}|f(x+y)-f(x)| d x \leq C|y|_{2}
$$

and this concludes the proof of Proposition 4.1.

Our second step in order to prove Theorem 1 is the next result.

Proposition 4.2. In $\mathbb{Z}_{2}$ we have the inclusion $B V\left(\mathbb{Z}_{2}\right) \subseteq \dot{B}_{1}^{1, \infty}\left(\mathbb{Z}_{2}\right)$.

Proof. Observe that we can characterize the Besov space $\dot{B}_{1}^{1, \infty}\left(\mathbb{Z}_{2}\right)$ by the condition

$$
\|f(\cdot+y)+f(\cdot-y)-2 f(\cdot)\|_{L^{1}} \leq C|y|_{2}, \quad \forall y \neq 0 .
$$

Let $f$ be a function in $B V\left(\mathbb{Z}_{2}\right)$, then we have

$$
\|f(\cdot+y)-f(\cdot)\|_{L^{1}} \leq C|y|_{2} .
$$

Summing $\|f(\cdot-y)-f(\cdot)\|_{L^{1}}$ in both sides of the previous inequality we obtain

$$
\|f(\cdot+y)-f(\cdot)\|_{L^{1}}+\|f(\cdot-y)-f(\cdot)\|_{L^{1}} \leq C|y|_{2}+\|f(\cdot-y)-f(\cdot)\|_{L^{1}}
$$

and by the triangular inequality we have

$$
\|f(\cdot+y)+f(\cdot-y)-2 f(\cdot)\|_{L^{1}} \leq C|y|_{2}+\|f(\cdot-y)-f(\cdot)\|_{L^{1}} .
$$


We thus obtain

$$
\|f(\cdot+y)+f(\cdot-y)-2 f(\cdot)\|_{L^{1}} \leq 2 C|y|_{2} .
$$

We have proved, in the setting of the 2 -adic group $\mathbb{Z}_{2}$, the inequalities

$$
C_{1}\|f\|_{\dot{B}_{1}^{1, \infty}} \leq\|f\|_{B V} \leq C_{2}\|f\|_{\dot{B}_{1}^{1, \infty}}
$$

so Theorem 1 follows.

\section{Improved Sobolev inequalities, $B V$ space and proof of Theorem 2}

We do not give here a global treatment of the family of inequalities of type (2); instead we focus on the next inequality

$$
\|f\|_{L^{2}}^{2} \leq C\|f\|_{B V}\|f\|_{\dot{B}_{\infty}^{-1, \infty}}
$$

and we want to know if this estimation is true in a 2-adic framework. Since in the $\mathbb{Z}_{2}$ setting we have the identification $\|f\|_{B V} \simeq\|f\|_{\dot{B}_{\infty}^{1, \infty}}$, the estimation (13) becomes

$$
\|f\|_{L^{2}}^{2} \leq C\|f\|_{\dot{B}_{1}^{1, \infty}}\|f\|_{\dot{B}_{\infty}^{-1, \infty}}
$$

This remark lead us to Theorem 2 which states that the previous inequalities are false.

Proof. We will construct a counterexample by means of the Littlewood-Paley decomposition, so it is worth to recall very briefly the dyadic bloc characterization of the norms involved in inequality (14). For the $L^{2}$ norm we have $\|f\|_{L^{2}}^{2}=\sum_{j \in \mathbb{N}}\left\|\Delta_{j} f\right\|_{L^{2}}^{2}$, while for the Besov spaces $\dot{B}_{1}^{1, \infty}$ and $\dot{B}_{\infty}^{-1, \infty}$ we have

$$
\begin{aligned}
\|f\|_{\dot{B}_{1}^{1, \infty}} & =\sup _{j \in \mathbb{N}} 2^{j}\left\|\Delta_{j} f\right\|_{L^{1}} \quad \text { and } \\
\|f\|_{\dot{B}_{\infty}^{-1, \infty}} & =\sup _{j \in \mathbb{N}} 2^{-j}\left\|\Delta_{j} f\right\|_{L^{\infty}} .
\end{aligned}
$$

We construct a function $f: \mathbb{Z}_{2} \longrightarrow \mathbb{R}$ by considering his values over the dyadic blocs and we will use for this the sets $Q_{j, k}$ defined in (7). First fix $\alpha$ and $\beta$ two non negative real numbers and $j_{0}, j_{1}$ two integers such that $0 \leq j_{0} \leq j_{1}$ with the condition

$$
2^{2 j_{0}} \leq \frac{\beta}{\alpha}
$$

Now define $N_{j}$ as a function of $\alpha$ and $\beta$ :

(15) $\quad N_{j}=2^{j} \quad$ if $0 \leq j \leq j_{0} \quad$ and $\quad N_{j}=\frac{\beta}{\alpha} 2^{-j} \leq 2^{j} \quad$ if $j_{0}<j \leq j_{1}$. 
and write

$$
\Delta_{j} f(x)=\left\{\begin{array}{lll}
\alpha 2^{j} & \text { over } & Q_{j+1,0}, \\
-\alpha 2^{j} & \text { over } & Q_{j+1,1}, \\
\alpha 2^{j} & \text { over } & Q_{j+1,2}, \\
-\alpha 2^{j} & \text { over } & Q_{j+1,3}, \\
& \vdots & \\
\alpha 2^{j} & \text { over } & Q_{j+1,2 N_{j}-2}, \\
-\alpha 2^{j} & \text { over } & Q_{j+1,2 N_{j}-1}, \\
0 & \text { elsewhere. }
\end{array}\right.
$$

Once this function is fixed, we compute the following norms

- $\left\|\Delta_{j} f\right\|_{L^{1}}=\sum_{k=0}^{N_{j}} \alpha 2^{j} 2^{-j}=\alpha N_{j}$,

- $\left\|\Delta_{j} f\right\|_{L^{\infty}}=\alpha 2^{j}$,

- $\left\|\Delta_{j} f\right\|_{L^{2}}^{2}=\sum_{k=0}^{N_{j}} \alpha^{2} 2^{2 j} 2^{-j}=\alpha^{2} 2^{j} N_{j}$,

and we build from these quantities the Besov and Lebesgue norms in the following manner:

1) For the Besov space $\dot{B}_{\infty}^{-1, \infty}$ :

$$
\|f\|_{\dot{B}_{\infty}^{-1, \infty}}=\sup _{0 \leq j \leq j_{1}} 2^{-j} \alpha 2^{j}=\alpha
$$

2) For the Besov space $\dot{B}_{1}^{1, \infty}$ :

By the definition (15) of $N_{j}$ we have $2^{j}\left\|\Delta_{j} f\right\|_{L^{1}}=2^{j} \alpha N_{j}=2^{2 j} \alpha$ if $0 \leq j \leq j_{0}$ and $2^{j}\left\|\Delta_{j} f\right\|_{L^{1}}=\beta$ if $j_{0}<j \leq j_{1}$. Since $2^{2 j_{0}} \leq \frac{\beta}{\alpha}$ we have:

$$
\|f\|_{\dot{B}_{1}^{1, \infty}}=\beta \text {. }
$$

3) For the Lebesgue space $L^{2}$ :

$$
\begin{aligned}
\|f\|_{L^{2}}^{2} & =\sum_{j=0}^{j_{1}} \alpha^{2} 2^{j} N_{j}=\sum_{j=0}^{j_{0}} \alpha^{2} 2^{2 j}+\sum_{j>j_{0}}^{j_{1}} \alpha^{2} 2^{j} \frac{\beta}{\alpha} 2^{-j}=\sum_{j=0}^{j_{0}} \alpha^{2} 2^{2 j}+\left(j_{1}-j_{0}\right) \alpha \beta \\
& =\alpha \beta\left(\frac{\alpha}{\beta} \sum_{j=0}^{j_{0}} 2^{2 j}+\left(j_{1}-j_{0}\right)\right) .
\end{aligned}
$$

With the condition $2^{2 j_{0}} \leq \frac{\beta}{\alpha}$, we obtain from the previous formula that

$$
\|f\|_{L^{2}}^{2} \simeq \alpha \beta\left(j_{1}-j_{0}\right)=\|f\|_{\dot{B}_{1}^{1, \infty}}\|f\|_{\dot{B}_{\infty}^{-1, \infty}}\left(j_{1}-j_{0}\right) .
$$

Thus, getting back to (14) and therefore to (13), we have for an universal constant $C$ the inequality

$$
\begin{aligned}
\|f\|_{\dot{B}_{1}^{1, \infty}}\|f\|_{\dot{B}_{\infty}^{-1, \infty}}\left(j_{1}-j_{0}\right) & \leq C\|f\|_{\dot{B}_{1}^{1, \infty}}\|f\|_{\dot{B}_{\infty}^{-1, \infty}} \\
\Longleftrightarrow\left(j_{1}-j_{0}\right) & \leq C,
\end{aligned}
$$

which is false since we can freely choose the values of $j_{1}$ and $j_{0}$. Theorem 2 is proved. 


\section{References}

[1] Y. Amice, Les nombres p-adiques, Presses Universitaires de France, Paris, 1975.

[2] J. Bergh and J. Löfstrom, Interpolation Spaces. An Introduction, Grundlehren der Mathematischen Wissenschaften, 223. Springer Verlag, 1976.

[3] D. Chamorro, Improved Sobolev Inequalities and Muckenhoupt weights on stratified Lie groups, J. Math. Anal. Appl. 377 (2011), no. 2, 695-09.

[4] - Some functional inequalities on polynomial volume growth Lie groups, Canad. J. Math. 64 (2012), no. 3, 481-496.

[5] A. Cohen, W. Dahmen, I. Daubechies, and R. De Vore, Harmonic Analysis of the space $B V$, Rev. Mat. Iberoamericana 19 (2003), no. 1, 235-263.

[6] P. Gérard, Y. Meyer, and F. Oru, Inégalités de Sobolev Précisées, Equations aux Dérivées Partielles, Séminaire de l'Ecole Polytechnique, exposé n IV (1996-1997).

[7] K. Ikeda, T. Kim, and T. K. Shiratani, On p-adic bounded functions, Mem. Fac. Sci. Kyushu Univ. Ser. A 46 (1992), no. 2, 341-349.

[8] L. C. Jang, T. Kim, J.-W. Son, and S.-H. Rim, On p-adic bounded functions. II, J. Math. Anal. Appl. 264 (2001), no. 1, 21-31.

[9] T. Kim, q-Volkenborn integration. Russ. J. Math. Phys. 9 (2002), no. 3, 288-299.

[10] N. Koblitz, p-adic Numbers, p-adic Analysis and Zeta-functions, GTM 58. Springer Verlag, 1977.

[11] M. Ledoux, On improved Sobolev embedding theorems, Math. Res. Lett. 10 (2003), no. 5-6, 659-669.

[12] E. M. Stein, Topics in Harmonic Analysis, Annals of mathematics Studies, 63. Princeton University Press, 1970.

[13] P. Strzelecki, Gagliardo-Nirenberg inequalities with a BMO term, Bull. Lond. Math. Soc. 38 (2006), no. 2, 294-300.

[14] V. S. Vladimirov, I. V. Volovich, and E. I. Zelenov, p-Adic Analysis and Mathematical Physics, World Scientific, Singapore, 1994.

Laboratoire d'Analyse et de Probabilités

UnIVERsité D'Evry VAL D'Essonne

Bâtiment I.B.G.B.I.

23 BD. DE FRANCE

91037 Evry Cedex-France, France

E-mail address: diego.chamorro@univ-evry.fr 